\title{
QUALIDADE DE ÁGUAS DE POÇOS DOS ASSENTAMENTOS DA CHAPADA DO APODI-RN PARA O USO NA AGRICULTURA
}

\author{
H. A. Oliveira ${ }^{1}$; H. N. Bezerra ${ }^{1}$; J. M. S. Araújo ${ }^{1}$; L. A. F. Tavares ${ }^{2}$ \\ ${ }^{1}$ Centro de Tecnologia - Universidade Federal do Rio Grande do Norte - herisonalves@yahoo.com.br - \\ helionogueirab@gmail.com - jurema.araujo@hotmail.com. \\ ${ }^{2}$ Departamento de Engenharia Rural - Universidade Estadual Paulista - leandrotavares@fca.unesp.br
}

Artigo submetido em março/2012 e aceito em fevereiro/2013

\section{RESUMO}

A agricultura é a principal fonte de renda das comunidades rurais, principalmente dos assentamentos localizados no estado do Rio Grande do Norte, sendo necessária a utilização de águas que garantam o desenvolvimento adequado das culturas. $O$ trabalho tem por objetivo analisar as águas de poços de assentamentos da Chapada do Apodi, classificando-as quanto ao seu uso na irrigação. Foram coletadas amostra de poços de 11 assentamentos para análises físico-químicas visando à classificação quanto aos critérios de qualidade de água segundo o UCCC e Richard. Observou-se que todos os poços apresentaram alguma restrição para o uso na irrigação sendo o poço 3 o que apresentou maior risco de salinidade e sodicidade do solo. Os poços 1, 8, 14 e 17 apresentaram um menor grau de restrição de acordo com o UCCC, enquanto que os poços 16, 18 e 19 apresentaram o menor risco de salinidade e sodicidade pela metodologia de Richards.

PALAVRAS-CHAVE: Salinidade, Irrigação, Comunidades rurais.

\section{ANALYSIS OF WELL WATER QUALITY FROM RURAL SETTLEMENT OF THE APODI-RN PLATEAU FOR USE IN AGRICULTURE}

\begin{abstract}
The agriculture is the main source of income for rural communities, especially in the settlements located in the state of Rio Grande do Norte, necessitating the use of water to ensure the proper development of crops. The study aims to analyze the well water of settlements of the Apodi Plateau, classifying them as to its use in irrigation. Samples were collected from wells of 11 settlements for physical and chemical analysis in order
\end{abstract}

to rank as the criteria for water quality according to the UCCC and Richard. It was observed that all wells showed some restraint for use in irrigation and the well 3 being that one who presented the greatest risk of soil salinity and sodicity. The wells $1,8,14$ and 17 had a lower degree of restriction in accordance with the UCCC, while the wells 16, 18 and 19 had the lowest risk of salinity and sodicity by the methodology of Richards.

KEY-WORDS: salinity, irrigation, rural communities. 


\section{QUALIDADE DE ÁGUAS DE POÇOS DOS ASSENTAMENTOS DA CHAPADA DO APODI-RN PARA O} USO NA AGRICULTURA

\section{INTRODUÇÃO}

Os assentamentos rurais no Brasil passaram a existir oficialmente a partir da década de 1980 (SILVA et al., 2010), ganhando ímpeto no decorrer da década de 90, tanto no campo quanto na cidade (BERGAMASCO, 1997). O Rio Grande do Norte, estado marcado pelas baixas condições financeiras da população rural, representa claramente a realidade da busca pela posse de terras, resultando na formação de assentamentos rurais, principalmente na Chapada do Apodi, que vem se destacado como um importante polo de exploração agrícola (MOTA et al., 2007) possuindo um dos mais importantes polos de irrigação do Nordeste (MENDES et al., 2010).

A Chapada do Apodi faz parte da Bacia Potiguar, onde as unidades estratigráficas estão representadas, predominantemente, pela formação calcária Jandaíra, com espessura média de 250 a 300m, no topo, e pelo arenito Açu, na base (MOTA et al., 2008). O clima é caracterizado por ser muito quente e semiárido, com a estação chuvosa se atrasando para o outono. A Chapada do Apodi apresenta pluviosidade média anual de 550 a $940 \mathrm{~mm}$ e a temperatura média anual é de $23^{\circ} \mathrm{C}$ (FIALHO, 2006).

Os assentamentos rurais são desenvolvidos por atividades de subsistência que necessitam de água (irrigação, dessedentação de animais e consumo humano), geralmente captada de poços. Na maioria das vezes essas atividades são executadas sem conhecimento adequado, devido à falta de estudo da população.

Além do clima árido e/ou semi-árido, da má drenagem e dos minerais primários, o manejo inadequado da irrigação pode gerar problemas de salinização do solos (CHAVES et al., 2006). Assim como a grande maioria das atividades humanas gera algum tipo de impacto negativo ao meio ambiente, a irrigação, se não for conduzida de modo racional, pode acarretar sérios danos ao solo, como o aumento das concentrações de sais e sódio trocável, que reduz sua fertilidade e, em longo prazo, pode provocar desertificação da área afetada (D'ALMEIDA et al., 2005). A salinidade diminui a disponibilidade de água às culturas, podendo reduzir a produção em até $50 \%$ (SANTANA et al., 2007).

A agricultura apresenta o maior consumo de água entre os setores da economia, sendo responsável por mais de $60 \%$ da demanda hídrica de boa qualidade (CARMO, 2007). Diante desse fator, torna-se necessário o uso de águas de qualidade marginal, entre elas as salinas, visando aumentar a oferta de água de boa qualidade para outros usos prioritários, como abastecimento humano. Partindo dessa problemática, surge a necessidade de desenvolvimento de estudos relacionados à utilização de águas salinas.

A análise das águas consiste na definição de sua composição físico-química, além da determinação dos riscos, quanto ao seu uso, para o homem e para o sistema solo-planta (salinização, sodicidade e toxidade). AYERS \& WESTCOT (1991), determinam alguns parâmetros que devem ser avaliados para irrigação com seus respectivos valores de referência, sendo estes: CE, Total de sólidos solúveis, RAS, Sódio, Cloro, Boro, Nitrogênio, Bicarbonato e o pH, entre outros.

A elaboração de trabalhos de detalhamento das disponibilidades de água dos mananciais (poços), considerando os aspectos sócio-econômicos, permite a obtenção de informações mais detalhadas sobre os recursos hídricos, visando um melhor planejamento e gerenciamento de 
projetos voltados para o uso das águas em suas diversas aplicações, promovendo uma conscientização com relação à utilização racional e, consequentemente, sustentável desses recursos.

Diante desse contexto, o trabalho tem por objetivo analisar as águas de poços dos assentamentos da Chapada do Apodi-RN, visando classificá-las quanto ao seu uso na irrigação, com intuito de servir de base para as comunidades em suas atividades de cultivo.

\section{MATERIAL E MÉTODOS}

O estudo foi desenvolvido em 11 assentamentos localizados na região da Chapada do Apodi, próximo ao município de Felipe Guerra. Foram coletadas amostras de $500 \mathrm{~mL}$ para cada poço de captação de águas desses assentamentos, em 03 de setembro de 2009, correspondente ao início do período seco da região para este ano, que apresentou precipitação acima da média, registrando-se chuvas até o mês de Julho.

A localização geográfica dos poços onde foram coletadas as amostras de água (Figura 1) foram georreferenciadas utilizando um GPS, com o auxílio do software GPS trackmaker e Google Earth.

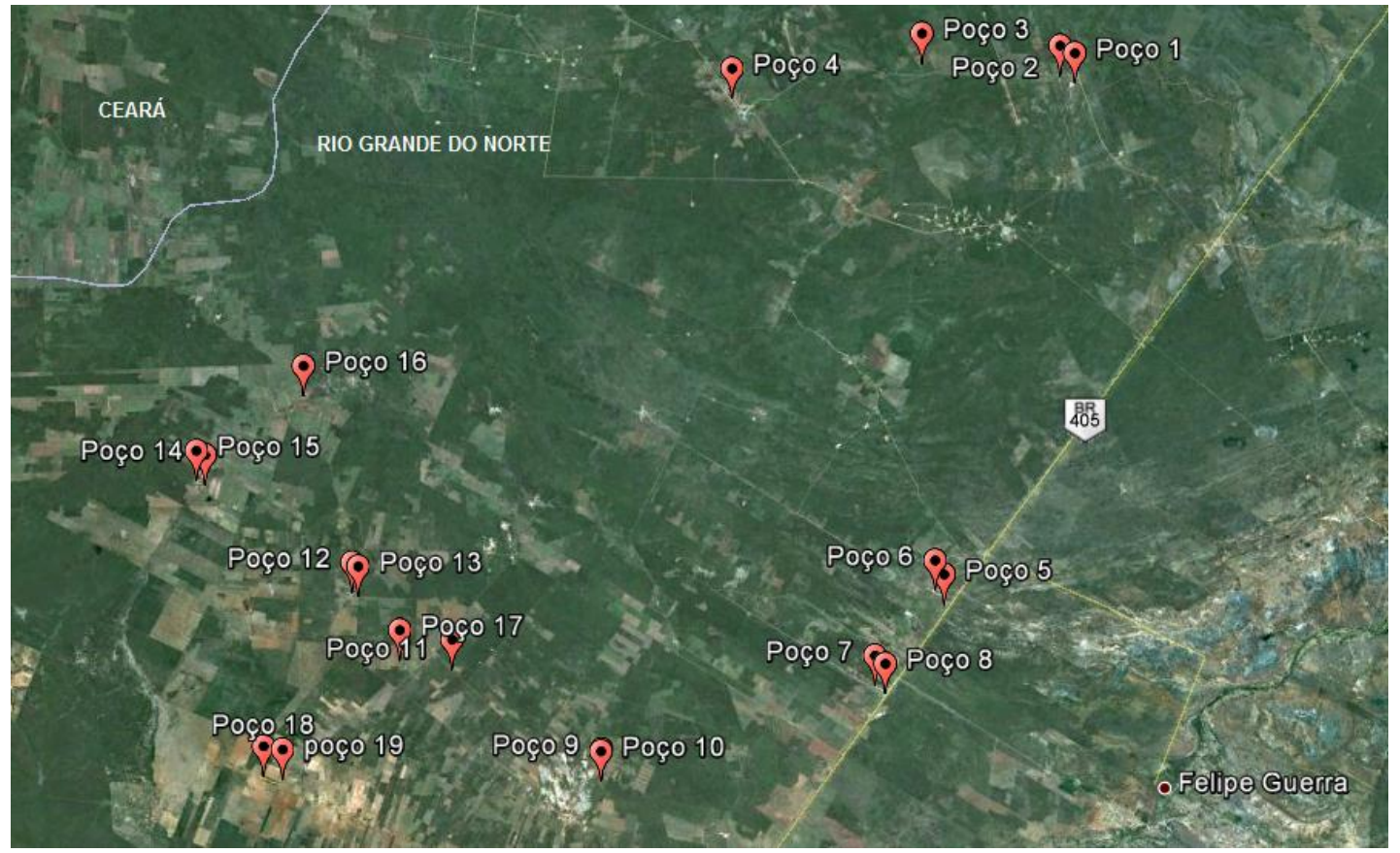

Figura 1 - Localização dos Poços.

Fonte: Google Earth (2012).

Para proceder as análises, foram coletadas apenas 1 amostra por poço, em garrafas plásticas de $500 \mathrm{~mL}$, lavadas 3 vezes com a água a ser coletada e rotuladas, contendo a identificação e características de cada poço.

As análises foram realizadas no Laboratório de Fertilidade do Solo e Nutrição de Plantas da UFERSA, onde foram determinados os parâmetros de acordo com metodologias adotadas pelo laboratório, sendo: $\mathrm{pH}$ e CE diretamente com leituras das amostras em Peagâmetro e Condutivímetro, respectivamente, previamente aferidos; Cálcio e Magnésio por titulometria com 
EDTA $(0,025 \mathrm{~N})$; Sódio e Potássio por fotometria em um aparelho denominado fotômetro de chama adequando-se as concentrações dos padrões e diluição das amostras em níveis compatíveis para as determinações; Carbonatos e Bicarbonatos por titulometria com ácido sulfúrico e o cloreto avaliado por volumetria de precipitação com nitrato de prata. A razão de adsorção de sódio (RAS) foi determinada conforme a metodologia descrita por AYERS \& WESTCOT (1999).

A partir dos resultados obtidos, as amostras de águas foram agrupadas em classes de salinidade e sodicidade avaliadas quanto ao grau de restrição de uso de acordo com as sugestões de valores de referência do Comitê dos Consultores da Universidade da Califórnia (UCCC, 1974) e classificadas pela metodologia proposta por RICHARDS (1954), sendo elaborado o gráfico dessa classificação com o auxílio do software QUALIGRAF.

Ainda foram coletadas informações com relação às principais utilizações das águas captadas em cada assentamento visitado (doméstico, humano, animal e horta), sendo registradas informações como profundidade dos poços, revestimento e localização geográfica, detalhadas na Tabela 1.

Tabela 1: Profundidade, uso e dados técnicos dos poços.

\begin{tabular}{ccccc}
\hline Poço & Assentamento & Profundidade $(\mathbf{m})$ & Revestimento & Uso \\
\hline 1 & Paulo Canapum & 280 & Ferro & Animal e Doméstico \\
2 & Paulo Canapum & 190 & Ferro & Animal e Horta \\
3 & Caiçara & 100 & PVC & Animal \\
4 & Sítio do Gois & 93 & PVC & Animal \\
5 & Frei Damião & 75 & Ferro & Humano e Animal \\
6 & Frei Damião & 225 & PVC & Humano e Animal \\
7 & São Manoel & 70 & PVC & Animal \\
8 & São Manoel & 380 & Ferro & Humano \\
9 & Vila Nova & 194 & PVC & Doméstico \\
10 & Vila Nova & 80 & PVC & Animal e Horta \\
11 & Aurora da Serra & 86 & Ferro & Animal \\
12 & Moacir Lucena & 96 & PVC & Animal e Horta \\
13 & Moacir Lucena & 36 & Ferro & Animal e Horta \\
14 & Paraíso & 180 & PVC & Animal e Horta \\
15 & Paraíso & 150 & Ferro & Humano \\
16 & Laje do Meio & 270 & PVC & Humano e Animal \\
17 & Aurora da Serra & 300 & Ferro & Humano e Animal \\
18 & Milagre & 180 & PVC & Horta \\
19 & Milagre & 180 & Ferro & Humano e Animal \\
\hline
\end{tabular}

\section{RESULTADOS E DISCUSSÕES}

A Tabela 2 mostra os resultados das variáveis físico-químicas das amostras coletadas. Os valores de $\mathrm{pH}$ variaram de 6,48 a 7,77, mostrando-se levemente ácida a alcalina. Para os níveis de sais dissolvidos, os valores da CEa variaram de 0,07 a 3,46 dS/m, apresentando águas de características doces $(42,1 \%)$, salobras $(52,6 \%)$ e salinas $(5,3 \%)$, conforme Figura 2 , baseado na estimativa dos Sólidos Totais Dissolvidos (STD), obtido pela multiplicação da condutividade elétrica por 0,65 , valor satisfatório para a região (Santos, 1997). O risco de sodicidade do solo foi determinado através do valor da RAS (Relação de Adsorção de Sódio), cujos valores variaram de 0,2 a $5,4(\mathrm{mmolC} / \mathrm{L}) 0,5$. 
Tabela 2: Variáveis físico-químicas da água dos poços.

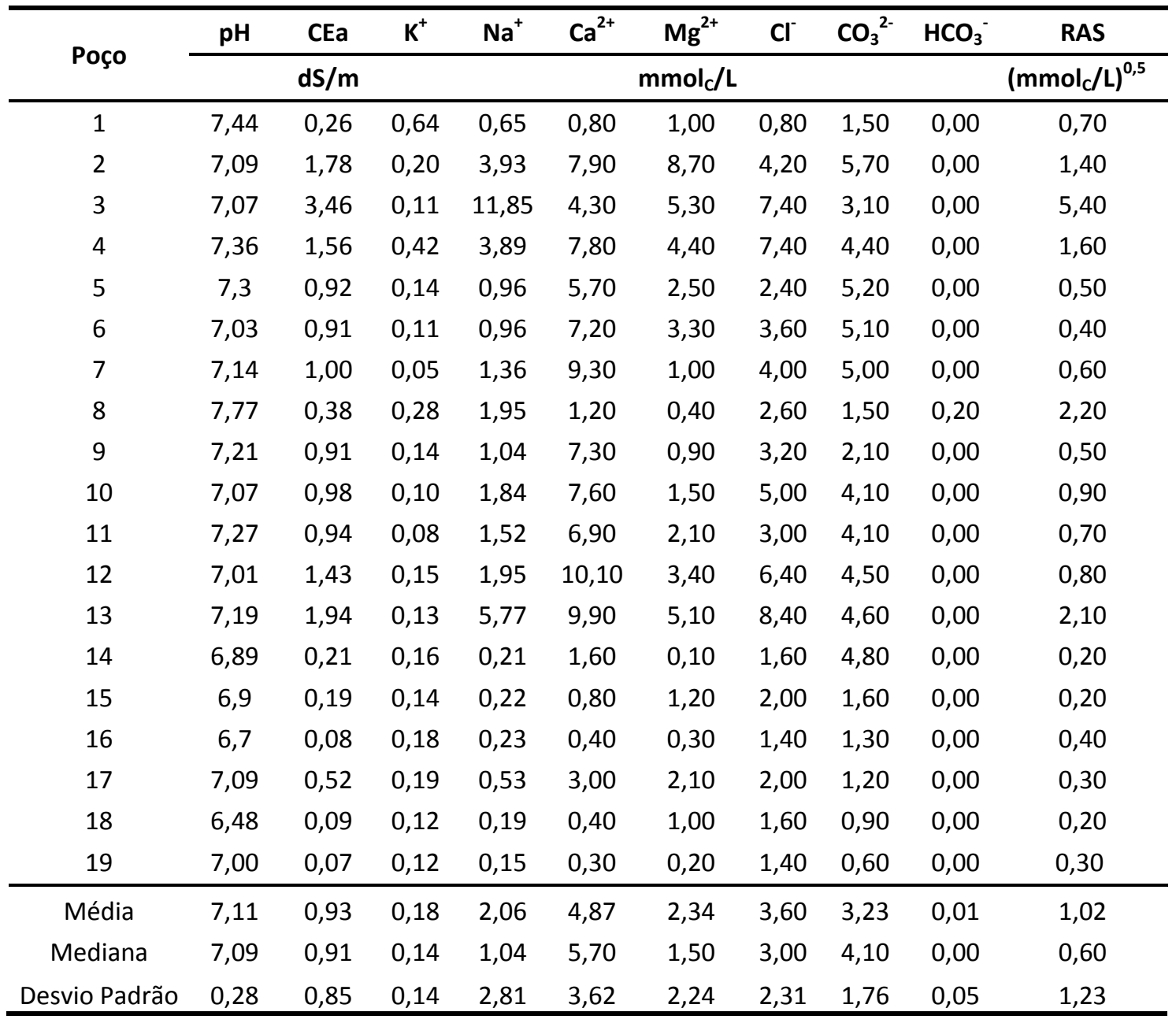

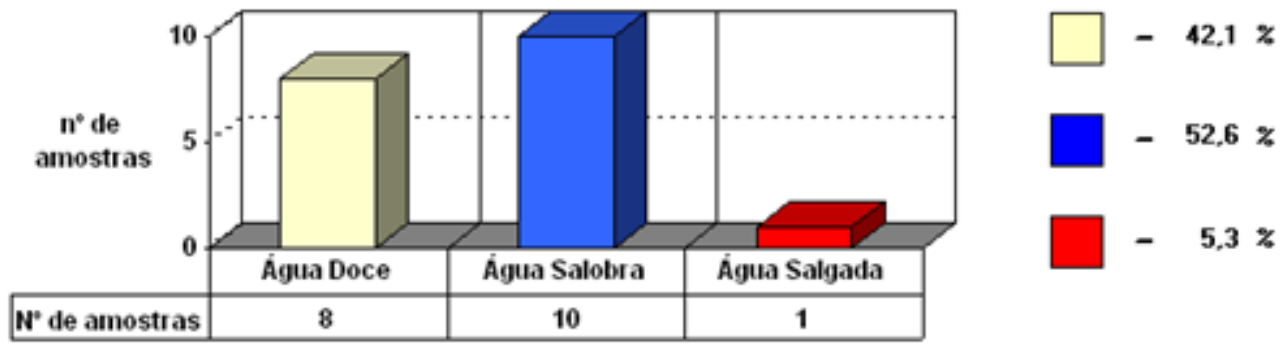

Figura 2 - Classificação da água quanto a sólidos totais dissolvidos.

Todas as amostras apresentaram valores de $\mathrm{pH}$ entre 6,5 a 8,4, intervalo recomendado para o uso na irrigação (FAO, 1974), exceto a amostra de água do poço 18 , que apresentou $\mathrm{pH}$ igual a 6,48 , podendo apresentar algum grau de restrição quanto ao uso. Em relação aos valores obtidos na análise da CEa, RAS, Cloreto e Sódio, o grau de restrição de uso para fins de irrigação segundo critérios do UCCC (1994) está apresentado na Tabela 3. 
Tabela 3: Grau de restrição de uso para irrigação (UCCC, 1974).

\begin{tabular}{|c|c|c|c|c|c|c|}
\hline \multirow{2}{*}{ Poço } & \multirow{2}{*}{ CEa } & \multirow{2}{*}{ RAS } & \multicolumn{2}{|c|}{ Cloreto } & \multicolumn{2}{|c|}{ Sódio } \\
\hline & & & IS & IA & IS & IA \\
\hline 1 & $\mathrm{~N}$ & LM & $\mathrm{N}$ & $\mathrm{N}$ & $\mathrm{N}$ & $\mathrm{N}$ \\
\hline 2 & LM & $\mathrm{N}$ & LM & LM & LM & LM \\
\hline 3 & $S$ & $\mathrm{~N}$ & LM & LM & $S$ & LM \\
\hline 4 & LM & $\mathrm{N}$ & LM & LM & LM & LM \\
\hline 5 & LM & $\mathrm{N}$ & $\mathrm{N}$ & $\mathrm{N}$ & $\mathrm{N}$ & $\mathrm{N}$ \\
\hline 6 & LM & $\mathrm{N}$ & $\mathrm{N}$ & LM & $\mathrm{N}$ & $\mathrm{N}$ \\
\hline 7 & LM & $\mathrm{N}$ & LM & LM & $\mathrm{N}$ & $\mathrm{N}$ \\
\hline 8 & $\mathrm{~N}$ & LM & $\mathrm{N}$ & $\mathrm{N}$ & $\mathrm{N}$ & $\mathrm{N}$ \\
\hline 9 & LM & $\mathrm{N}$ & $\mathrm{N}$ & LM & $\mathrm{N}$ & $\mathrm{N}$ \\
\hline 10 & LM & $\mathrm{N}$ & LM & LM & $\mathrm{N}$ & $\mathrm{N}$ \\
\hline 11 & LM & $\mathrm{N}$ & $\mathrm{N}$ & LM & $\mathrm{N}$ & $\mathrm{N}$ \\
\hline 12 & LM & $\mathrm{N}$ & LM & LM & $\mathrm{N}$ & $\mathrm{N}$ \\
\hline 13 & LM & $\mathrm{N}$ & LM & LM & LM & LM \\
\hline 14 & $\mathrm{~N}$ & LM & $\mathrm{N}$ & $\mathrm{N}$ & $\mathrm{N}$ & $\mathrm{N}$ \\
\hline 15 & $\mathrm{~N}$ & $S$ & $\mathrm{~N}$ & $\mathrm{~N}$ & $\mathrm{~N}$ & $\mathrm{~N}$ \\
\hline 16 & $\mathrm{~N}$ & $S$ & $\mathrm{~N}$ & $N$ & $\mathrm{~N}$ & $\mathrm{~N}$ \\
\hline 17 & $\mathrm{~N}$ & LM & $\mathrm{N}$ & $\mathrm{N}$ & $\mathrm{N}$ & $\mathrm{N}$ \\
\hline 18 & $\mathrm{~N}$ & $S$ & $\mathrm{~N}$ & $\mathrm{~N}$ & $\mathrm{~N}$ & $\mathrm{~N}$ \\
\hline 19 & $\mathrm{~N}$ & $S$ & $\mathrm{~N}$ & $\mathrm{~N}$ & $\mathrm{~N}$ & $\mathrm{~N}$ \\
\hline
\end{tabular}

$\mathrm{N}$ - Nenhuma restrição; LM - Ligeira a Moderada; S - Severa restrição; IS - Irrigação por Superfície e IA - Irrigação por Aspersão.

Em relação à CEa, a amostra do poço 3 foi a única a apresentar uma severa restrição quanto ao uso, enquanto as demais variaram entre nenhuma a moderada restrição. Quanto ao valor da RAS, apenas os poços 15, 16, 18 e 19 apresentaram uma restrição severa.

Para o Cloreto e o Sódio, as amostras apresentaram valores semelhantes, onde a maioria apresentaram de nenhuma a moderada restrição, sendo apenas o poço 3 a apresentar severa restrição para método de irrigação por superfície para o Sódio.

De uma forma geral, o poço 3 apresentou o maior grau de restrição quanto ao uso para irrigação, já os poços 1, 8, 14 e 17 apresentaram um comportamento inverso, com o menor grau de restrição entre todos os poços, por possuírem as maiores profundidades em regiões de afloramento do Calcário Jandaíra.

Na Tabela 4 verifica-se a classificação da água da irrigação quanto ao risco de salinidade e sodicidade conforme diagrama proposto por RICHARDS (1954). As águas dos poços variaram entre as classes COS1 (riscos de salinidade nulo e de sodicidade baixo), C1S1 (riscos de salinidade baixo e de sodicidade baixo), C2S1 (riscos de salinidade médio e de sodicidade baixo), C3S1 (riscos de salinidade alto e de sodicidade baixo) e C4S2 (riscos de salinidade muito alto e de sodicidade médio), nas proporções, respectivamente, de 15,8,10,5,15,8,52,6 e 5,3\% das amostras das águas dos poços avaliados. 
Tabela 4: Classificação segundo Richards (1954).

\begin{tabular}{cccc}
\hline Poço & Classificação & Poço & Classificação \\
\hline 1 & C2 S1 & 11 & C3 S1 \\
2 & C3 S1 & 12 & C3 S1 \\
3 & C4 S2 & 13 & C3 S1 \\
4 & C3 S1 & 14 & C1 S1 \\
5 & C3 S1 & 15 & C1 S1 \\
6 & C3 S1 & 16 & C0 S1 \\
7 & C3 S1 & 17 & C2 S1 \\
8 & C2 S1 & 18 & C0 S1 \\
9 & C3 S1 & 19 & C0 S1 \\
10 & C3 S1 & & \\
\hline
\end{tabular}

De acordo com a classificação proposta por RICHARDS (1954) o poço 3 também apresentou alto risco de salinidade e sodicidade do solo, podendo causar danos as culturas, conforme mostra a Figura 3. Para o uso desta água na irrigação será necessário o cultivo de espécies tolerantes a salinidade e que possuam uma boa absorção desses sais, a fim de reduzir os riscos de salinização e sodificação do solo. Já os poços 16, 18 e 19 apresentaram menores riscos a salinidade e sodicidade do solo, sendo adequado para qualquer cultura.

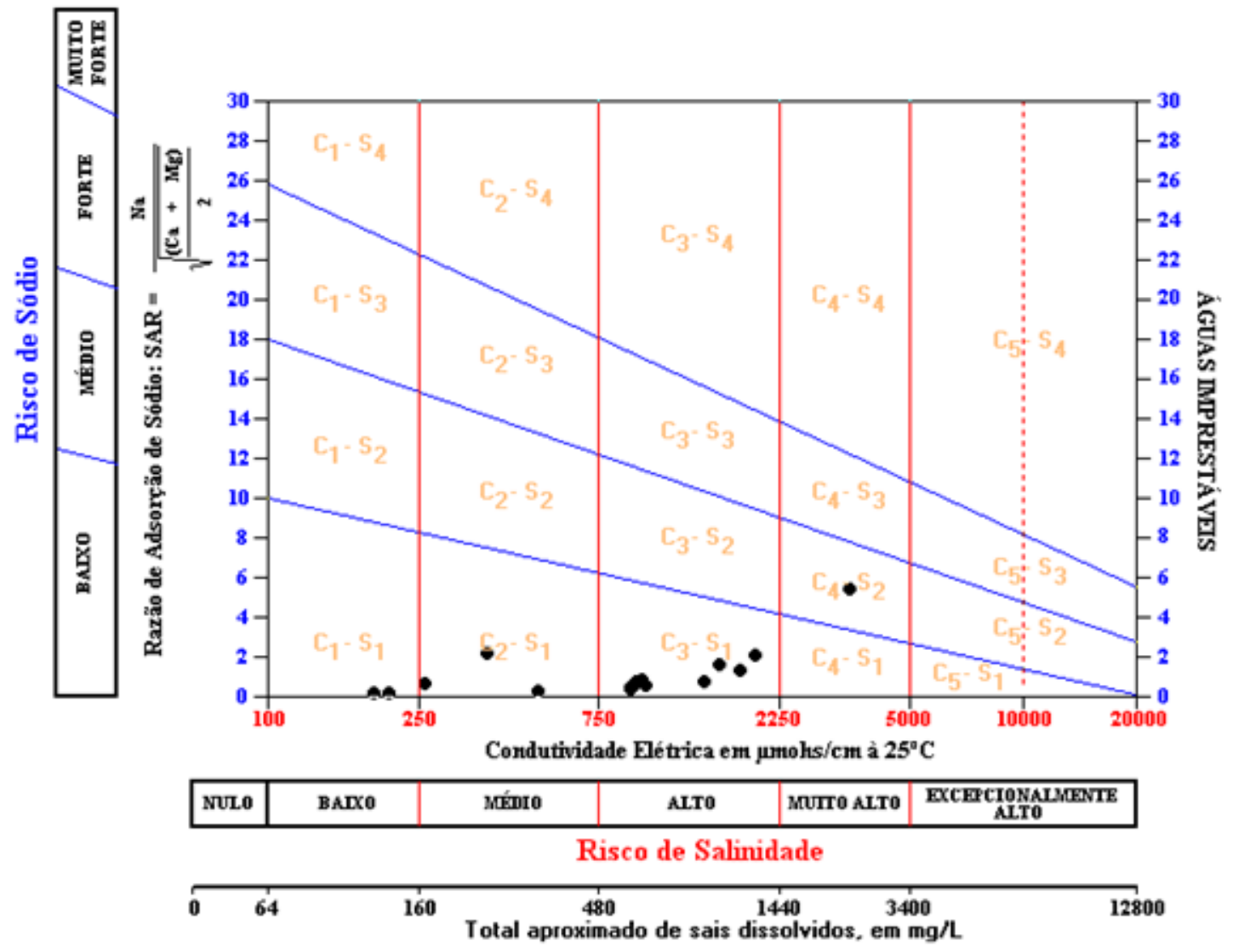

Figura 3 - Classificação da água para irrigação - Richards (1954). 
É importante destacar que os valores podem alterar de um período para outro, ou seja, apresentar resultados diferentes das concentrações de sais entre os períodos de chuva e de seca, decorrente da diminuição do volume de água dos mananciais na estação seca e aumento na estação chuvosa, sendo necessária a realização de uma nova coleta no final do período seco, a fim de fazer uma comparação entre os resultados.

\section{CONCLUSÕES}

Todos os poços apresentaram alguma restrição para o uso na irrigação, mas, na maioria dos casos, não comprometendo a sua utilização, sendo necessária a verificação da sensibilidade da cultura a ser irrigada quanto ao nível de salinidade e sodicidade da água.

O poço 3 apresentou o maior risco de salinidade e sodicidade do solo, necessitando de um manejo adequando quando for utilizado para irrigação.

Os poços 1, 8, 14 e 17 apresentaram um menor grau de restrição para o uso na irrigação pelos critérios do UCCC (1994), enquanto que os poços 16, 18 e 19 foram os que apresentaram o menor risco de salinidade e sodicidade pela metodologia de Richards.

No Rio Grande do Norte não há legislação e/ou normativo usado na classificação da qualidade da água para irrigação, portanto utilizam-se padrões internacionais de restrição de uso desse recurso na prática agrícola, necessitando assim de estudos para a criação de padrões de qualidade da água para o estado.

\section{REFERÊNCIAS BIBLIOGRÁFICAS}

1. AYERS, R.S.; WESTCOT, D.W. A qualidade de água na agricultura. 2.ed. Campina Grande: UFPB, 1999, 153p.

2. BERGAMASCO, S. M. P. P. A realidade dos assentamentos rurais por detrás dos números. Estud. av., São Paulo, v. 11. 1997.

3. CARMO, R. L.; OJIMA, A. R. L. O.; OJIMA, R.; NASCIMENTO, T. T. Água virtual, escassez e gestão: o Brasil como grande exportador de água. Ambient. soc., Campinas, v. 10, n. 2, Dec. 2007.

4. CHAVES, L. C. G.; ANDRADE, E. M.; CRISOSTOMO, L. A.; LOPES, J. F. B.; NESS, R. L. L. Risco de degradação em solo irrigado do distrito de irrigação do perímetro Araras Norte, Ceará. Revista Ciência Agronômica, v. 37, 2006. p. 292-298.

5. D'ALMEIDA, D. M. B. A.; ANDRADE. E. M.; MEIRELES, A. C. M.; NESS, R. L. L. Importância relativa dos íons na salinidade de um cambissolo na chapada do Apodi, Ceará. Eng. Agríc., Jaboticabal, v. 25, n. 3, Dec. 2005. 
6. FAO. Development and management of water resources, Jamaica. Rio Minho. Annex III Water Quality. FAO Report No. FAO, Rome. 1974

7. FIALHO, J. S.; GOMES, V. F. F.; OLIVEIRA, T. S.; SILVA JÚNIOR, J. M. T. Indicadores da qualidade do solo em áreas sob vegetação natural e cultivo de bananeiras na chapada do Apodi-CE. Revista Ciência Agronômica, v. 37, 2006. p. 250-257.

8. MENDES, A. M. S.; DUDA, C. P.; NASCIMENTO, C. W. A.; LIMA, J. A. G.; MEDEIROS, A. D. L. Acúmulo de metais pesados e alterações químicas em cambissolo cultivado com meloeiro. Rev. bras. eng. agríc. ambient., Campina Grande, v. 14, n. 8, 2010.

9. MOTA, J. C. A.; ASSIS JÚNIOR, R. N.; AMARO FILHO, J.; ROMERO, R. E.; MOTA, F. O. B.; LIBARDI, P. L. Atributos mineralógicos de três solos explorados com a cultura do melão na chapada do Apodi: RN. Rev. Bras. Ciênc. Solo, Viçosa, v. 31, n. 3, 2007.

10. MOTA, J. C. A.; ASSIS JÚNIOR, R. N.; AMARO FILHO, J.; LIBARDI, P. L. Algumas propriedades físicas e hídricas de três solos na chapada do Apodi, RN, cultivados com melão. Rev. Bras. Ciênc. Solo, Viçosa, v. 32, n. 1, Feb. 2008.

11. RICHARDS, L. A. Diagnosis and improvement of saline and alkali soils. Washington: U.S, Department of Agriculture, 160p. 1954.

12. SANTANA, M. J.; CARVALHO, J. A.; SOUZA, K. J.; SOUSA, A. M. G.; VASCONCELOS, C. L.; ANDRADE, L. A. B. Efeitos da salinidade da água de irrigação na brotação e desenvolvimento inicial da cana-de-açúcar (Saccharum spp) e em solos com diferentes níveis texturais. Revista Ciência Agrotécnica, v.31, 2007. p.1470-1476.

13. SANTOS, A. C. NOÇÕES DE HIDROQUÍMICA. IN: FEITOSA, F. A. C. \& MANUEL FILHO, J..Hidrogeologia: conceitos e aplicações. CPRM, LABHID-UFPE. Cap. 5. 1997. p. 81-108.

14. SILVA, E. B.; NOGUEIRA, R. E.; UBERTI, A. A. A. Avaliação da aptidão agrícola das terras como subsídio ao assentamento de famílias rurais, utilizando sistemas de informações geográficas. Rev. Bras. Ciênc. Solo, Viçosa, v. 34, n. 6, Dec. 2010.

15. UCCC-University of California Committee of Consultants. Guidelines for interpretation of water quality for agriculture. Davis: University of California, 13p. 1974. 